\title{
Assessment of Attitudes, Skills and Source of Knowledge on Utilization of EBP Among Registered Nurses in Xiangya Hospital of Central South University, Changsha, China
}

\author{
Boka Dugassa Tolera ${ }^{1,2,{ }^{*}}$, Feng Hui ${ }^{2}$ \\ ${ }^{1}$ School of Nursing, Addis Ababa University, Addis Ababa, Ethiopia \\ ${ }^{2}$ Xiangya School of Nursing, Central South University, Changsha, China \\ Email address: \\ bokadugassa@gmail.com (B. D. Tolera), feng.hui@csu.edu.cn (Feng Hui) \\ ${ }^{*}$ Corresponding author
}

\section{To cite this article:}

Boka Dugassa Tolera, Feng Hui. Assessment of Attitudes, Skills and Source of Knowledge on Utilization of EBP Among Registered Nurses in Xiangya Hospital of Central South University, Changsha, China. American Journal of Nursing Science. Vol. 6, No. 2, 2017, pp. 99-112. doi: 10.11648/j.ajns.20170602.14

Received: January 5, 2017; Accepted: January 16, 2017; Published: February 20, 2017

\begin{abstract}
Background: Evidence-Based Practice (EBP) is one of the nursing professional roles that can lead them to provide the best and more effective quality patient care. However, little studies are available across china on registered nurses' attitude, skills and utilization of knowledge source for implementation of evidence based practice. Due to this the extent to which evidence based practice being utilized and implemented in nursing practice is still in its infancy across China. Objectives: The aim of this study was to assess the attitude, skills and source of knowledge on implementation of EBP among registered nurses working at selected teaching hospitals. Method: A descriptive institutional based cross-sectional study was conducted to assess nurses' attitude, skills and utilization of knowledge source for implementation of EBP. A total of 366 RN were participated in filling self-administered questionnaire. The descriptive and inferential statistical analysis was performed by using SPSS version 20. The cut point for level of statistical significance was set at $P<0.05$ and all tests were 2-sided. Result: Overall, registered nurses who were participated in this study had a positive attitudes towards utilization of evidence based practice $(\mathrm{M}=3.53, \mathrm{SD}=$ 0.97). However, the level of skill in performing different $\mathrm{EBP}$ activities $(\mathrm{M}=2.82 \mathrm{SD}=1.34)$ and the use of knowledge source to support daily care practice $(M=3.01, S D=0.98)$ were found to be low. Similarly, participants indicated that they use more human and printed resources than electronic resources for supporting their practice (mean $=3.69, \mathrm{SD}=1.01$; mean $=2.88, \mathrm{SD}=$ 0.80 ; and mean $=2.57, \mathrm{SD}=1.05$ ) respectively. Conclusion: It is recommended that organizational and nursing educational support were needed to improve this gap through providing training, creating awareness, and enhancing nurses' abilities for utilization and implementation of evidence based practice. Additionally, it is recommended that further research is needed to identify the influence of individual and organizational factors on implementation of evidence based nursing practice.
\end{abstract}

Keywords: Registered Nurse, Evidence Based Practice, Attitude, Skills, Source of Knowledge

\section{Introduction}

As health care professionals, the term evidence-based practice is common to registered nurses. It is described as " a life-long problem-solving approach to the delivery of health care that integrates the best evidence from well-designed studies with a patient's preferences, values and a clinician's expertise, which includes internal evidence gathered from patient information" [1]. This approach help the nurses to critically assess and use all information resources in order to correctly identify clinical problem, apply the most high-quality intervention, and re-evaluate the outcome of their performance for future improvement [2]. In addition, It assist changes in nursing professional roles with updated current knowledge to identify main clinical issues, reduce medication errors and increase nurses' self-confidence in performing different EBP activities [3]. Hence, utilization of EBP is considered as a mark of excellence for professional nurses including registered nurses [4].

Moreover, best evidence based nursing practice refers to 
methodologically sound, clinically relevant research about effectiveness and safety of nursing interventions for quality of patient outcome [5]. This indicates that EBP is a process that begins with research and ends with practice. Therefore, in order to effectively apply evidence in practice, in addition to skills in taking a history and conducting the five nursing process (assessment, diagnosis, planning, implementation and evaluations), nurses must have the ability to: (a) identify the main clinical issue/ problems, (b) formulate clinically relevant questions; (c) conduct online searches (using databases and Web search engines), (e) relate research finding to their clinical practice and point out similarities and differences; (d) apply an intervention based on the most applicable evidence and (f) evaluate the application of intervention and identify areas of improvement [6]. Such skills may vary among registered nurses and influenced by several factors including nurses' level of education, year of work experience, nurses' attitudes, self-efficacy, nurses' awareness to use up-to-dated knowledge source and their leaders' or organizational support [7]. However, the need of our patient should be considered as critical issue in any health care organization around the world.

Therefore, currently, it has been a global priority for health care organizations to improve patient outcomes, and quality and consistency of care through utilization of evidence based practice knowledge and skills [3]. However, not all healthcare professionals and managers use EBP in daily care practice [8]. This indicates, most of our patients do not receive care in accordance with the latest scientific evidence [9]. Practicing health care in the absence of up to date knowledge is risky and a threat to patient safety [10]. For example, the approach of evidence-based nursing Practice requires nurses to integrate technical skills and professional knowledge with up-to-date scientific evidence to apply evidence-based nursing care plans to solve the patients' problems [1]. However, a considerable gap still exists between utilization of up-to-dated scientific evidence and the current nursing practice [11].

In fact, implementing EBP is challenging, because it depends on the willingness of the individual staff nurses and senior leaders or managers to embrace EBP in a changing health care environment that is based on scientific evidence [12] For example, International Council of Nurses (ICN) noted that "In the era of evidence based practice and knowledge-driven health care, nurses are constantly challenged to discover new and better ways of delivering care that is grounded in new knowledge and evidence derived through research" [13]. Lack of technical skills in conducting online search, lack of problem-solving skills in making recommendations for practice changes, inability to adapt to changing environments were cited as a major barrier for nurse to integrate evidence into their daily practice [14]. Thus, most nurse do not use electronic resources such as online databases including CINALH, Pub Med and Cochrane library very often to keep their knowledge and skill and to adopt EBP [15]. They tend to seek professional information from human resources such as colleagues, doctors, superiors and other healthcare providers rather than using journal articles or textbooks [16].
Evidence has suggested that for many health professionals in low- and middle-income countries, the concept of evidence-based practice approaches are new [17]. Only a small percentage of nurses' integrate and use EBP in their daily care practice. Hence, the application of research-based evidence in nursing practice is limited [18]. For example a report from Italy revealed that only $41.4 \%$ of nurses read scientific journals or guidelines, $37 \%$ incorporate EBP into practice [19], In Poland only $42 \%$ of nurses use scientific evidence more often in their everyday nursing practice [20]. Similarly, evidence suggest that most research activities in African countries are linked to educational or academic programmers and in many African countries, it is not clearly known how much nurses use research evidence to improve their practice in order to ensure high quality of patient care [21]. However, there is a few evidence suggesting poor utilization of scientific evidence at work place in some African countries. For instance, the study done in Kenya found that only $20.6 \%$ of the nurses use research evidence in their work [22], another study from Ethiopia revealed that only $15.7 \%$ of nurses use evidence based Practice [23].

As a developing country, China is no exception to this poor use of EBP. For example, research has shown that the evidence-based practice progress in nursing profession is not well embraced in china [24]. It is better developed and has a longer history in the medical disciplines, especially for physicians and other health technicians who are deployed at tertiary hospitals [25]. Consequently, most Chinese people prefer to visit tertiary hospitals due to better medical technology and perceived technical quality of the provider [26]. Thus, the burden of care which is now on the tertiary level has led to the rising costs [27]. A large amounts of money are spent annually on high-quality health service [28]. One major objective behind all these efforts is to help doctors, and medical technicians in provision of the best possible care and treatment to patients [29].

Similar to other western country, the Chinese registered nurse work in direct care of patients; assessing patients' needs and making decisions on nursing interventions [30]. However, evidence suggests that nurses in China are still facing a number of significant challenge in utilization and implementation of Evidence based practice [31]. Most Chinese nurses still could not get the up-to- date and high-quality scientific evidences efficiently and conveniently [32]. Majority of them were not familiar with the concept of EBP, and still they are utilizing the traditional ways of working, and incorporated research findings into clinical practice infrequently [33]. Consequently, Chinese patients rely heavily on treatments from traditional Chinese medicine: including herbal treatments and diet therapy [32]. This is may be due to inadequate knowledge and skills, weak beliefs about the value of EBP, poor attitudes toward EBP and lack of awareness for using up- to- dated EBP resource. For instance, there is a little empirical research published measuring the level of attitude, skill and use of knowledge source for implementation of EBP among registered nurses across china.

Therefore, measuring the level of attitudes, skill and 
utilization of knowledge source for implementation of EBP among registered nurses is extremely important to enhance quality of health care service across china. Moreover, the Chinese Ministry of health clearly encourage the integration of appropriate evidence into the activity of all health care professionals including nurses. Because the approach of EBP indicates not only better patient care, but also tremendous change in health care management at an economic and professional level [34]. It can improve the quality and costs of healthcare along with patient outcomes as well as reduces unnecessary variation of care [35]. It provides opportunity for nurses to ensure that clinical interventions and decisions making processes are safe and suitable for every patient group and enabling effectiveness in patient advocacy [36]. it helps nurses provide high-quality patient care based on research knowledge [37]. So, enhancing the knowledge and skill of EBP will help nurses to see the value of EBP [38]. Thus, the finding of these study has significant implications for staff nurse, leaders/ managers, and educators who are in a key positions to build a supportive culture for EBP and to provide the time, educational skills and resources which is necessary for all nurse to implement evidence-based care.

Therefore, the purpose of this study was to assess the attitudes, level of skill and utilization of source of knowledge on implementation of EBP among registered nurses working at selected teaching hospitals in Changsha City, Hunan province

\section{Conceptual Frame-Work}

Globally, several organizational models have been proposed that assist nurses in the utilization and implementation of evidence based practice. These are the John Hoptins, Evidence- Based Practice Model, Iowa model, Rosswurm and Larabee's Model and the Advancing Research and clinic practice through close collaboration (ARCC) Model. For this study the conceptual framework was developed after review of several literatures that is done in different part of the world on factors influencing the utilization and implementation of EBP. Moreover, The Advancing Research and Clinical Practice through Close Collaboration (ARCC) model was used to guide this study. According ARCC model, an assessment of the organizational readiness for system-wide implementation of EBP is considered as the first step. Additionally, In this model, registered nurses working as clinical mentors with in-depth knowledge of and skills in EBP is considered as one of key professional personnel to bring the individual behavior change and organizational culture change through implementation of EBP [39]

\section{Study Objectives, Research Questions and Research Hypothesis}

\subsection{General Objectives}

The general objective of this study was to assess the attitude, skills and source of knowledge on implementation of EBP among registered nurses working at selected teaching hospitals of Central South University, Changsha, Hunan province.

\subsection{Specific Objectives}

The specific objective of this study were to:

1. explore registered nurses' self-reported attitude towards utilization of EBP in Xiangya hospital of CSU.

2. describe registered nurse' self-reported skills on implementation of EBP in Xiangya hospital of CSU.

3. identify registered nurses' source of knowledge for utilization of EBP in Xiangya hospital of CSU

4. describe factors influencing registered nurses on utilization of Evidence based practice (EBP)

\subsection{Research Questions}

This study addresses the following research questions:

(a) What are registered nurses' attitudes towards utilization of EBP?

(b) What are registered nurses skill in performing different EBP activities?

(c) What are registered nurses' source of knowledge for utilization of EBP?

(d) What are factors affecting nurses in utilization of evidence based practice?

\subsection{Research Hypothesis}

(1) Are there any differences between attitude, skills and utilization of knowledge source for registered nurses based on their educational level?

(2) Are there any differences between attitude, skills and utilization of knowledge source for registered nurses based on their destination/position?

(3) Are there any differences between attitude, skills and utilization of knowledge source for registered nurses based on their year of work experience?

\section{Methodology}

\subsection{Study Setting and Population}

This descriptive, cross-sectional study design was conducted on October 01 to October 30/ 2016 in Xiangya specialized teaching hospital of Central South University (3082 bedded), which is the largest teaching hospital in Hunan province, South China. It serves as a referral center for patients from surrounding primary health care facilities as well as other secondary healthcare facilities within the province. Many Medical students and nurses undergo their training (in both under graduate and post graduate training) in this institution.

Data from human resource management indicates that more than 2.5 million patients visit this hospital per year (including the outpatient and inpatient department). At the time of data collection, there were approximately 2551 qualified registered 
nurses employed at the Xiangya referral and teaching hospital of Central South University. The ratio of nurse to doctors was 2.1:1. A total of 5100 employees were employed in this institution.

\subsection{Instrument}

This self-reported Evidence-based Practice Questionnaire was developed by (Majid et al., 2011) on adoption of evidence-based practice in clinical decision making among nurses [15]. The questionnaire was previously shown to be a valid and reliable instrument [15]. This instrument has been used in several country to examine nurses' perception on Evidence based practice. For example, In addition to initially used in Singapore [15], this questionnaire has used in Nigeria [40], in Ethiopia [23] and twice used in Iran [7]. Permission was obtained to use this self-reported evidence-based practice questionnaires from Authors. A forward backward translation approach from English Version into Mandarin Chinese Version was applied in accordance with the World Health Organization's procedures for translation and adaptation before data collection started [42]

Then before data collection, the Chinese versions questionnaire was reviewed by an experts who were highly qualified health professional consisting of five professors as well as researchers, on its appropriateness and relevance (by using two sets of rating scales, ranging from 1-not appropriate/relevant to 4-very appropriate/relevant) to the clinical settings. Then all of the items on the attitude and skills/practice scale were rated as content and linguistic relevant and appropriate (i.e., 3 or 4), except two items on section four (i.e. use of knowledge source) needing minor amendments. After that the content and face validity of the self-reported EBPQ were again confirmed by 5 professors/ experts in the field of EBP and the Scale-Content Validity Index (S-CVI) was 0.97. Thus, It was used to assess the accuracy of the items to the research objectives and the literature that guided the items in the instrument.

To establish the reliability and stability, the questionnaire was pilot tested (before data collection), in a different but similar hospital outside of the study area with 30 registered nurses selected randomly. The Chinese version has established a very good face, content, and construct validity with internal consistency reliabilities of Cronbach's alpha coefficient more than 0.80 .

The content of questionnaire was divided into four sections assessing different categories designed to address a particular construct of Evidence Based Practice (EBP) as follows:

Section 1: Consists 8 questions on nurse's socio-demographic information, including sex, age, highest nursing qualification, destination, working department, employment status and familiarity with EBP

Section 2: examined the nurses' attitudes towards utilization of EBP. A set of five items were used to assess the nurses' attitude (opinions) towards utilization of EBP by using a 5 -point Likert-type scale rated from 1-5 (1= strongly agree and $5=$ strongly disagree). Then the Chinese version has established acceptable face, content, and construct validity with internal consistency reliabilities and Cronbach's alpha coefficient $(\alpha=0.76)$

Section 3: Assessed a self-reported skill in EBP. We used nine items responded in 5-point Likert scale ranging from 1 to 5. A response of "poor" received a score of 1 and a response of "excellent" received a score of 5. The Chinese version has established a very good face, content, and construct validity with internal consistency reliabilities and Cronbach's alpha coefficient $(\alpha=0.86)$

Finally, section four explored the use of knowledge source for supporting practice, it was rated from never (1) to always (5). The utilization of sources of knowledge are divided into three dimensions, (1) Print knowledge sources covered by six items (e.g. Frequency of utilizing research articles/journals, textbooks, handout and pamphlets) (2) Electronic knowledge sources covered by nine items (e.g. nursing e-books, medical databases, online tutorials, blogs on EBP, different website providing up-to dated informations etc.) and (3) Human knowledge sources covered by seven items. (E.g. Ward colleagues, supervisor, doctors and nursing managers etc,). A total of 22 item was used to explore the use of knowledge source for supporting practice. The Chinese version has indicated a very good face, content, and construct validity with internal consistency reliabilities and Cronbach's alpha coefficient $(\alpha=0.82)$. In addition, the reliability of overall questionnaire was evaluated using Cronbach's alpha coefficient ( $\alpha=0.88)$. For more information (See table 6 )

In general, we used the baseline for decision and interpretation of our result (i.e. after summing the scores and calculating the mean values). Then the mean scores of bellow 3.2, 3.3 up to 3.4 and 3.5 and above were considered as low, moderate and favorable respectively.

\subsection{Sampling Method}

Sampling is a process of selecting a portion of the designated population to represent the entire population [43]. The purpose of sampling is to study a sub-group of a population in order to make generalizations of the population from which the group was drawn [44]. Therefore, in this study, data were collected from hospital departments by using simple random sampling method. There were a total of six special departments in this hospital: (1) Inpatient Department (2) Out Patient Department (3) Emergence Department (4) Operation Room (5) ICU and (6) Others (for nurses employed in administration office). Since each of those selected hospital departments have different number of registered nurses, the obtained sample size (366) were proportionally allocated to each departments. From the selected department, there were a largest number of registered nurses employed at inpatient department (1694). So a stratified random sampling method was adopted to select participants. First, all inpatient department was divided in to two (i.e. Surgical unit and Medical unit). Then, 24 inpatient departments were randomly selected and a total number of 81 registered nurse were taken from surgical unit and 81 of them were taken from medical unit by lottery method. Also a total of 17 registered nurses were taken from administrative office by using lottery 
method. Finally, a total of 366 study participants were randomly selected from each department. (IPD $=162$, ICU $=$ $63, \mathrm{OPD}=39, \mathrm{EMD}=52, \mathrm{OR}=33$, Others $($ in $\mathrm{AO})=17)$.

\subsection{Data Collection Technique}

For quality of data collection, five nurses who have experience in data collection were recruited and trained for data collection. To enhance the response rate, the questionnaires attached to the cover letter were hand delivered to the head nurses in each department. Then registered nurses who were randomly selected from the staff lists of the hospital department were invited to complete the questionnaire, consisting of the self-reported attitude and skills in EBP, use of knowledge source for practice and the demographic information sheet. They were informed to participate in the study during a shift change (during handover at morning time) by the investigator, and via announcement letter (flyers) posted on the unit, by the head nurses of each departments. They were informed the survey would take approximately less than 20 minutes of the participants' time and they could complete the survey at the hospital.

The hard copy of questionnaires were enclosed in postal envelope and were distributed to the selected respondents to be filled. In addition, a self-addressed envelope was provided for the nurses to deposit the completed questionnaires into a collection box on their ward. The contact telephone number of one of the researchers was included in the letter in case the nurses needed to ask questions about the study. Completed questionnaires were checked every day by principal investigator and supervisors. Then a total of $(n=366)$ potential respondents were participated in the study, including bedside registered nurses(staff nurse) and nursing managers or leader who were employed at nursing administration office.

\subsection{Inclusion and Exclusion Criteria}

\subsubsection{Inclusion Criteria}

Inclusion criteria are a set of conditions that must be met for a respondent to be included in the sample [43]. Therefore, in this study, all qualified RNs, from all shifts who are working as clinicians, or as clinical instructors or tutors (mentors) with an experience of more than one year \& above were included.

\subsubsection{Exclusion Criteria}

The followings are exclusion criteria for any participant to be excluded in the study.

(1) Nurses with seriously ill during the time of data collection will excluded.

(2) All students (under and post graduate) were excluded from the study.

(3) All nurses who are unwilling to participate were excluded.

(4) All nurses having less than 1 year work experience were excluded.

\subsection{Sample Size Determination}

Using a 95\% confidence interval (two sided), $\alpha=0.05$ margin of error and $10 \%$ is also added to compensate for non-response rate and other contingencies. Since no previous study was found, 0.5 prevalence was used.

$$
\begin{aligned}
& n=\frac{(Z \alpha / 2)^{2} p(1-p)}{d^{2}} \\
& n=\frac{(1.96)^{2}(0.5 * 0.5)}{(0.05)^{2}}=384
\end{aligned}
$$

Since the study population were 2551 which is below 10,000, we used correction formula as follow:

$$
\mathrm{Nc}=\mathrm{n} /(1+\mathrm{n} / \mathrm{N})
$$

$\mathrm{Nc}=384 /(1+384 / 2551)=333$. By adding $10 \%$ nonresponse rate, the final sample size,

$\mathrm{nf}=333+33=366$ study subject were required for the study.

\subsection{Data Analysis}

The completed questionnaires were examined daily for completeness and properly completed questionnaires were entered into computer and analyzed using IBM, SPSS version 20.0, Window 8. Items from sections 2 to 4 of the questionnaire were ranked for each section in order of importance after calculating means for each item. Descriptive statistics (frequencies, percentages, mean and standard deviation) for each item were calculated, and the correlation between each item in each section (perceived attitudes, skills and use of source of knowledge) was calculated. Independent t-test was also used to make comparison among groups in the study. The respondents were grouped depending on their socio-demographic information gender, age, highest educational level (diploma, bachelor and master degree), position (staff nurse and leader/ manager) and work experience as RN (1-4years, 5-9 years and 10 years and above) and familiarity with EBP (Yes or No). The cutoff for level of statistical significance was set at $P<0.05$ and all tests were 2-sided.

\subsection{Ethical Considerations}

Before the study begin, the ethical approval for the study was obtained from Ethical Review Committee (ERC) of the Central South University, Xiangya School of Nursing. The obtained official letters was delivered to Xiangya hospital of CSU. In addition, a letter explaining about the purpose, method and anticipated benefit and risk of the study were attached to each questionnaire. Informed consent were taken orally from each participant before start of data collection. Confidentiality was assured by indicating they are not requested to write their name on the questionnaire and by assuring that their responses will not in any way be linked to them.

\section{Result}

\subsection{Demographic Characteristics}

In total, 366 registered nurses from different specialized 
care units were involved in filling the questionnaires. All of them returned the questionnaires representing a $100 \%$ response rate. However, 3 questionnaires were incompletely filled. Ninety- seven percent $(n=356)$ of respondents were female; $3 \%(n=10)$ were male. About half of the respondents' age (50\%) was ranged between 20-29 years, 34\% of respondents' age was reported in between 30-39 years old. Nearly $12 \%$ of the respondents held master's degrees, with $54 \%$ had a bachelor degree and 34\% held diploma. Approximately $95 \%$ of the respondents described themselves as staff nurses, and nearly $5 \%$ described themselves as leaders /managers. Most respondents were employed in an Inpatient department (44\%) followed by ICU department (17\%), OPD (11\%), Operation room (9\%), EMD (14\%) and administration office (5\%). Majority of respondents (45\%) had work experience 10 years and above. Most respondents $(60 \%)$ indicated that they are not familiar with EBP (see table 1).

Table 1. Demographic characteristics of the respondents $(n=366)$.

\begin{tabular}{ll}
\hline Variable & N $(\%)$ \\
\hline 1. Sex & $10(2.7)$ \\
Male & $356(97.3)$ \\
Female & \\
2. Age & $181(49.5)$ \\
20-29 years & $124(33.9)$ \\
30-39 years & $52(14.2)$ \\
40-49 years & $9(2.5)$ \\
50years and above & $126(34.4)$ \\
3. highest qualification & $198(54.1)$ \\
Diploma & $42(11.5)$ \\
Bachelor degree in nursing & \\
Master degree in nursing & $349(95.4)$ \\
4. Destination/Position & $17(4.6)$ \\
Staff nurse & \\
Manager/Leader & \\
\hline
\end{tabular}

\begin{tabular}{ll}
\hline Variable & N $(\%)$ \\
\hline 5. Working department & $162(44.3)$ \\
Inpatient & $63(17.2)$ \\
ICU & $39(10.7)$ \\
OPD & $33(9.0)$ \\
Operation Room & $52(14.2)$ \\
Emergency Department & $17(4.6)$ \\
Others & \\
6. Nursing experience & $73(19.9)$ \\
1-4Yrs & $130(35.5)$ \\
5-9 Yrs. & $163(44.5)$ \\
10 Yrs. and above & \\
7. Employment Status & $347(94.8)$ \\
Official Full time worker & $19(5.2)$ \\
Part time worker & $147(40.2)$ \\
8. Familiarity with EBP & $219(59.8)$ \\
Yes & \\
No
\end{tabular}

\subsection{Attitude Towards Utilization of EBP}

In order to assess nurses' perceived attitude towards utilization of EBP, a set of five items responded in five Likert scale were used. In this study, the items' scores 1 to 2 , which is responded in strongly agree and agree were combined and represented as negative items. And the items' score 4 to 5, which is responded in strongly disagree and disagree were combined and represented as positive item. Then the highest score and the most positive one for attitude was for the item "Most research articles are not relevant to my daily practice." $3.89 \pm 0.90)$. While the positive rate of it was $76.2 \%$. The lowest score and most negative one for attitude was for item "My workload is too high to keep up-to-date with all new evidences" $(2.89 \pm 0.96)$, while the positive rate for this item was only $25.9 \%$ (see table 2 ).

Table 2. Scores of self-reported attitude in each items of $E B P Q(N=366)$.

\begin{tabular}{lcc}
\hline Items & Mean \pm SD & $\begin{array}{c}\text { \% of responding in 4-5 response } \\
\text { rate }\end{array}$ \\
\hline Most research articles are not relevant to my daily practice & $3.89 \pm 0.90$ & 76.2 \\
I don't like people questioning my clinical practices which are based on established methods & $3.74 \pm 0.80$ & 69.9 \\
I believe evidence-based practice has only limited utility. & $3.72 \pm 0.91$ & 66.7 \\
I prefer using more traditional methods instead of changing to new approaches. & $3.42 \pm 1.30$ & 61.2 \\
My workload is too high to keep up-to-date with all new evidences & $2.89 \pm 0.96$ & 25.9 \\
Total & $3.53 \pm 0.97$ & 3 \\
\hline
\end{tabular}

* The Mean and SD (Standard deviation) is calculated out 5. (Ranging from $1=$ strongly agree, $5=$ strongly disagree)

A comparisons between attitude sub scale and socio-demographic data was performed. A moderate positive correlation was observed between self-reported attitude sub scale and highest nursing qualification $(\mathrm{r}=0.40, \mathrm{p}<0.001)$, nursing position $(\mathrm{r}=0.18, \mathrm{P}=0.001)$, work experience $(\mathrm{r}=0.17$, $\mathrm{P}=0.001)$, working $\operatorname{department}(\mathrm{r}=0.24, \mathrm{P}<0.001)$, employment status $(\mathrm{r}=-0.11, \mathrm{P}=0.03)$ and familiarity with $\operatorname{EBP}(\mathrm{r}=-0.45, \mathrm{P}<0.001)$. Indicating that nurses had a favorable attitude towards utilization of EBP.

The statistical test has shown that there were significant difference between the mean score of each items in attitude sub scales based on highest nursing qualification: Diploma and bachelor degree $(\mathrm{t}=-5.93, \mathrm{p}<0.001)$, bachelor and masters $(\mathrm{t}=-4.38, \mathrm{p}<0.001)$, diploma and master degree $(\mathrm{t}=$ $-7.38, \mathrm{p}<0.001)$. Similarly, the mean score of items in attitude sub scale for master degree nurses $(4.08 \pm 0.52)$ were more than bachelor degree nurses $(3.64 \pm 0.61)$ and diploma nurse $(3.17 \pm 0.74)$. This indicates that master degree nurses had much positive attitude towards utilization of EBP than bachelor degree and diploma nurse. A

Statistical significant difference was also observed between staff nurses and nurse leaders/ manager $(\mathrm{t}=-3.49, \mathrm{P}=0.001)$, ICU and Inpatient department $(\mathrm{t}=-4.36, \mathrm{p}<0.001)$ and nurses attitude based on familiarity with $\operatorname{EBP}(\mathrm{t}=9.84, \mathrm{p}<0.001)$. No statistically significant differences were found between attitudes and other demographic variables $(\mathrm{P}>.05)$ 


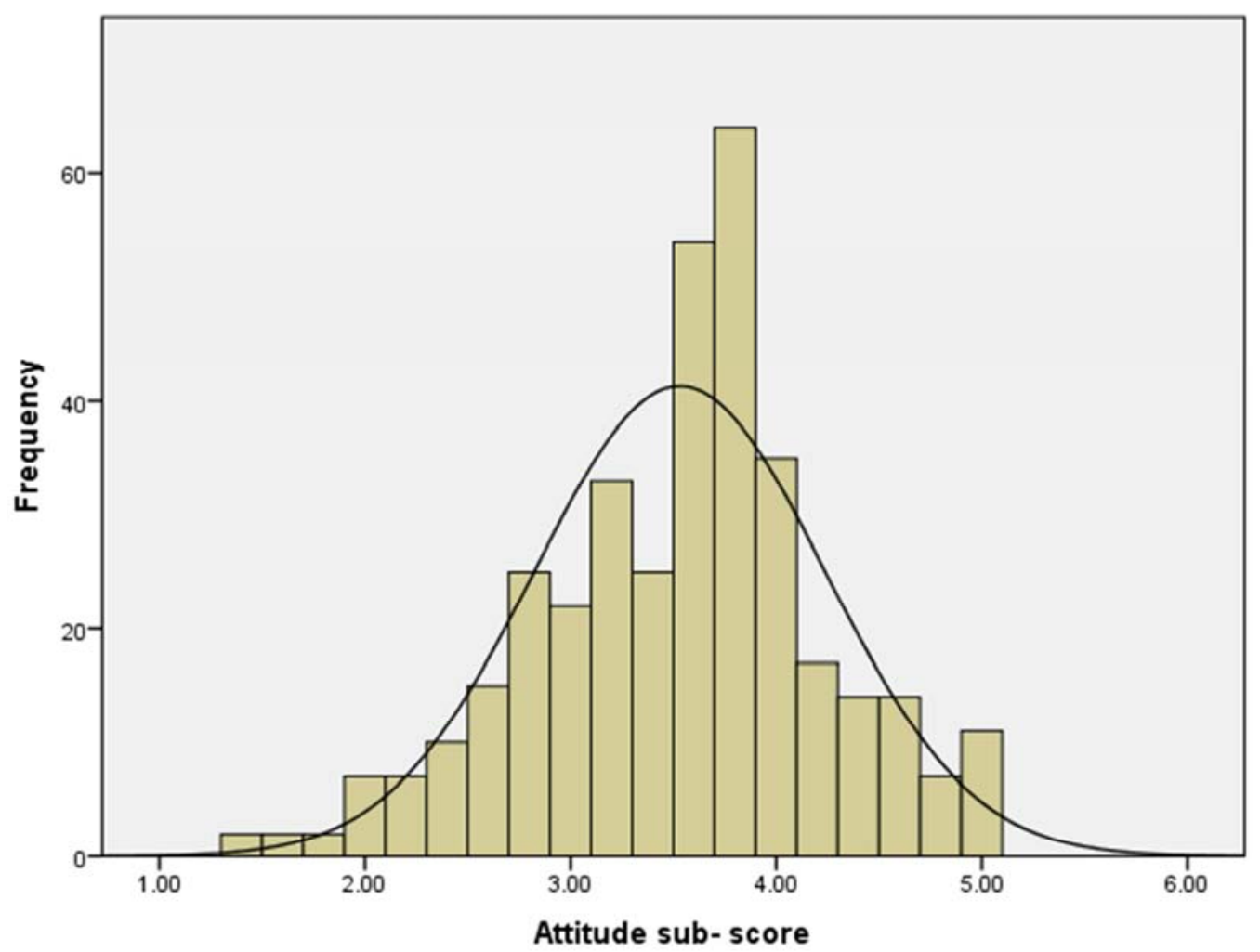

Figure 1. Attitude sub-scores. Mean $(S D)=3.53(0.97)$, Range $=5.00-1.40 ;$ Median $=3.60$.

\subsection{Skills in EBP}

In this sub scale, the item scores of 1 to 2 were combined and represented as a negative items. And the item scores of 4 to 5 were combined and represented as a positive item. A participants mean scores was ranged from 2.16 to 3.48 on a scale of 1 to 5 , with 5 being the optimal score (see table 3). As can be seen the total mean score and Standard deviation (SD) for self-reported skill in EBP sub scale was 2.82 and 1.34 respectively. For the majority of the skill items, more than half of respondents having master degree were indicated a high level ('4' and '5') of self-reported skill in EBP. However, a vast number of participants having diploma and bachelor degree were rated their skills in between Poor to good ((' 1 ' and ' 3 '). Then the item with the lowest mean score was "read a research report and have a general notion about its strength and weaknesses" $(2.16 \pm 1.35)$. It was also the most negative one in skills-subscale, with $66.1 \%$ negative rate. While, the item with the highest mean score was "Identify the main clinical issue/ problems" $(3.48 \pm 1.10)$. It was also the most Positive one in self -reported skills subscale, with only $24.1 \%$ negative rate. In addition, respondents had moderate skill in conducting online search using search engine $(2.67 \pm 1.37)$. They also indicated that a vast majority of them (60.1\%) were unable to use check list to assess research articles (see table 3)

Table 3. Scores of self-reported skills in each items of EBPQ $(N=366)$.

\begin{tabular}{|c|c|c|c|}
\hline I am able to: & Mean \pm SD & \%responding 1-2 & Rank \\
\hline Read a research report and have a general notion about its strength and weaknesses & $2.16 \pm 1.35$ & 66.1 & 1 \\
\hline Evaluate the application of intervention and identify areas of improvement & $2.25 \pm 1.38$ & 62.9 & 2 \\
\hline Use check list to assess research articles & $2.36 \pm 1.43$ & 60.1 & 3 \\
\hline Conduct online searches (using databases and Web search engines). & $2.67 \pm 1.37$ & 47.6 & 4 \\
\hline Apply an intervention based on the most applicable evidence & $2.77 \pm 1.43$ & 46.4 & 5 \\
\hline Distinguish between different types of questions & $3.10 \pm 1.44$ & 31.1 & 6 \\
\hline Relate research finding to my clinical practice and point out similarities and differences & $3.29 \pm 1.32$ & 29.2 & 7 \\
\hline Identify the main clinical issue/ problems & $3.48 \pm 1.10$ & 24.1 & 9 \\
\hline Total score & $2.82 \pm 1.34$ & & \\
\hline
\end{tabular}

* The Mean and SD (Standard deviation) is calculated out 5 response rate (i.e. 1 Poor 5=Excellent)

A comparisons between skill sub-scales and demographic variable was performed. A weak positive Spearman's correlation was observed between self-reported skills and nursing highest qualification $(\mathrm{r}=0.32, \mathrm{p}<0.001)$, nursing position $(\mathrm{r}=0.20, \mathrm{P}<0.001)$, Work experience $(\mathrm{r}=0.32$, $\mathrm{P}<0.001)$, Working department $(\mathrm{r}=0.21, \mathrm{P}<0.001)$ and age $(\mathrm{r}$ $=0.31, \mathrm{P}<0.001)$

Additionally, a strong statistical significant difference was 
observed between diploma and master degree nurses in response to skill in EBP items $(\mathrm{t}=-9.93, \mathrm{p}<0.001)$ bachelor and master degree $(\mathrm{t}=-10.97, \mathrm{P}<0.001)$. The mean scores of self-reported skill in EBP for master degree nurses (4.01 \pm 1.01) were much higher than bachelor degree nurses $(2.72 \pm$ $1.32)$ and diploma nurse $(2.59 \pm 1.29)$. This means nurses who had a master's degree were likely to be more confident in performing different EBP activities than diploma and bachelor degree nurses. Further, a statistical significant difference was observed between participants having work experience 1-4 years and 10 years and above $(\mathrm{t}=-5.44, \mathrm{p}<0.001), 5$-9year and 10 year and above $(\mathrm{t}=-4.99, \mathrm{P}<0.001)$. This means nurses with longer work experience were likely to be more confident in implementing EBP activities than those having shorter work experience. Additionally, a statistical significant difference has shown between staff nurse and nurse manager $(\mathrm{t}$ $=-4.49, \mathrm{P}<0.001)$, Inpatient and $\mathrm{ICU}(\mathrm{t}=-4.13, \mathrm{P}<0.001)$, Inpatient and OPD $(\mathrm{t}=-4.01, \mathrm{P}<0.001)$. Besides, significant difference was observed based on familiarity with evidence based practice $(\mathrm{t}=5.33, \mathrm{p}<0.001)$. No statistically significant differences were found between self-reported skills and other demographic variable $(\mathrm{P}>.05)$.

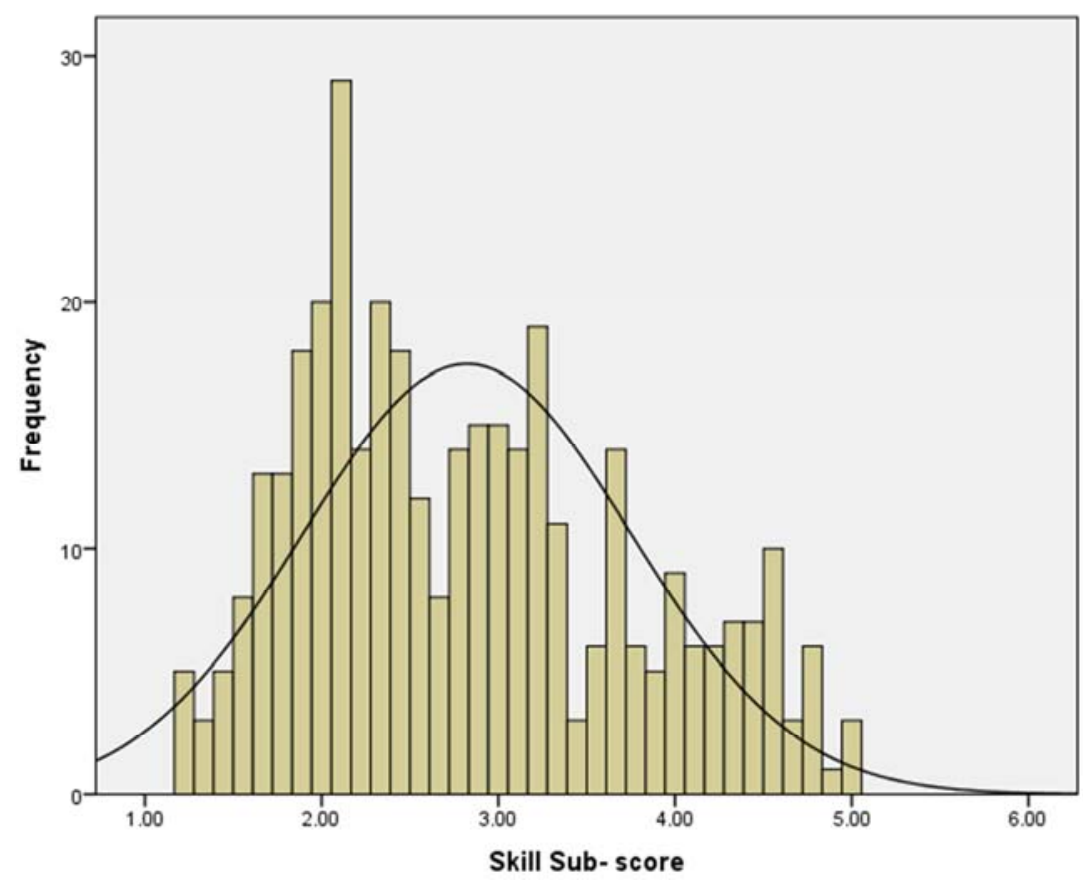

Figure 2. Skill sub-score: Mean $(S D)=2.82(1.34)$, Median $=2.67$, Range $=5.00-1.22$.

\subsection{Use of Knowledge Source for Supporting Practice}

In current study, the mean and standard deviation of sub-score for the use of knowledge source are 3.01 and 0.98 respectively (See table 4). As can be seen, the finding of our study shows that more than half of the participants $(55.8 \%)$ never or rarely use scientific journal articles. Additionally, a large proportion of participants $(57.1 \%)$ did not use medical database like CINAHL as a source of information/knowledge and about $(55.4 \%)$ respondents did not use blog on EBP. However, a vast majority of respondents use human information source (ward colleagues, supervisor and doctors) for evidence in supporting their daily practice (See table 4).

Table 4. Scores of self-reported Source of Knowledge for supporting daily practice in each items of EBPQ $(N=366)$.

\begin{tabular}{|c|c|c|c|}
\hline Print Knowledge Sources & Mean \pm SD & $\%$ in $1-2$ response rate & Rank \\
\hline Journal articles & $2.60 \pm 0.89$ & 55.8 & 1 \\
\hline Newspapers & $2.67 \pm 0.68$ & 38.0 & 2 \\
\hline Pamphlets/handouts (produced by healthcare companies, hospitals) & $2.79 \pm 0.75$ & 36.0 & 3 \\
\hline Reference books (e.g. medical dictionaries, encyclopedias) & $2.89 \pm 0.59$ & 20.2 & 4 \\
\hline Textbooks & $3.44 \pm 0.72$ & 3.6 & 5 \\
\hline \multicolumn{4}{|l|}{ Electronic Knowledge Sources } \\
\hline Medical databases (e.g. CINAHL) & $2.33 \pm 0.95$ & 57.1 & 1 \\
\hline Digital medical and nursing libraries & $2.49 \pm 0.97$ & 53.5 & 3 \\
\hline Online tutorials provided by professional associations, medical libraries, foreign hospitals & $2.50 \pm 1.13$ & 50.0 & 4 \\
\hline websites providing information about a specific medicine, treatment or symptom & $2.58 \pm 1.05$ & 49.0 & 5 \\
\hline Up to Date; MD Consult & $2.64 \pm 0.96$ & 45.7 & 6 \\
\hline Electronic Standard of Procedures (i.e. Work instructions, support) & $2.65 \pm 1.07$ & 42.3 & 7 \\
\hline Nursing e- books & $3.00 \pm 1.16$ & 34.5 & 8 \\
\hline \multicolumn{4}{|l|}{ Human Knowledge Sources } \\
\hline
\end{tabular}




\begin{tabular}{llll}
\hline Print Knowledge Sources & Mean \pm SD & \% in 1-2 response rate & Rank \\
\hline Nursing research committee/ EBN Group & $3.34 \pm 1.29$ & 36.9 & 2 \\
Nursing management staff & $3.65 \pm 1.24$ & 27.3 & 3 \\
Doctors & $3.89 \pm 1.05$ & 12.6 & 4 \\
Nursing supervisor & $4.16 \pm 0.99$ & 9.8 & 5 \\
Ward Colleague & $4.27 \pm 0.89$ & 4.4 & 6 \\
Total & $3.01 \pm 0.98$ & & \\
\hline
\end{tabular}

* The Mean and SD (Standard deviation) is calculated out 5 response rate (i.e. 1 Never 5= Always)

A comparisons between source of knowledge sub-scales and demographic variable was performed. A weak positive correlation has shown between source of knowledge sub scale and educational qualification( $\mathrm{r}=.022, \mathrm{P}<0.001)$, Work position $(\mathrm{r}=0.23, \mathrm{P}<0.001)$, Work experience $(\mathrm{r}=0.11, \mathrm{P}$ $=0.03)$ and working department $(\mathrm{r}=0.25, \mathrm{P}<0.001)$. On other hand, a strong statistically significant difference was observed between diploma and master degree nurses $(\mathrm{t}=-6.10, \mathrm{P}<$ $0.001)$, bachelor and master degree nurses $(\mathrm{t}=-5.73, \mathrm{P}<0.001)$.
Similarly, a significant difference was observed between Staff nurse and nurse manager $(\mathrm{t}=-4.98, \mathrm{P}<0.001)$, indicating that nurses managers/leaders were more likely to use formal sources of knowledge in the form of research journals and other up-to-dated source of information than staff nurses. Interestingly, nurses having work experience 10 years and above made greater use of information gained via the electronic source than nurses having work experience between 1 - 4 year and $(t=-2.21, P=0.03)$.

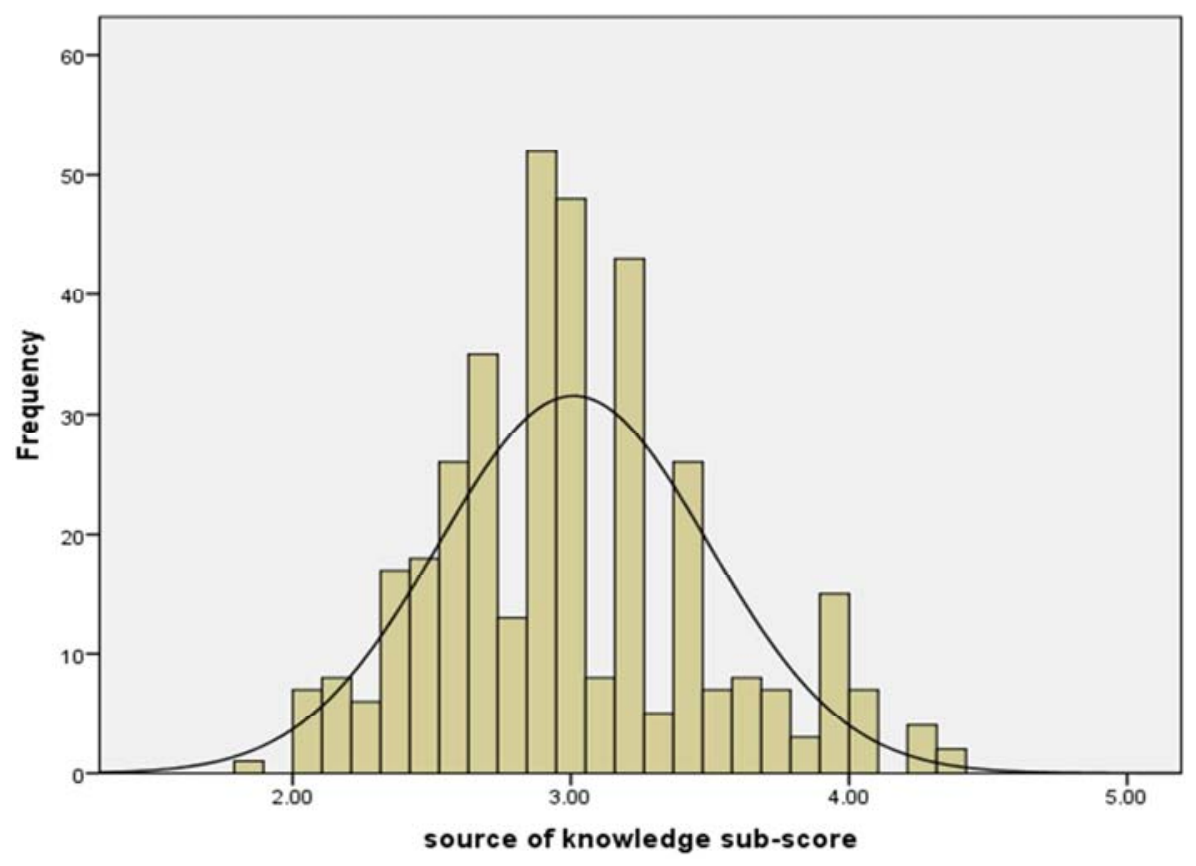

Figure 3. Source of Knowledge Sub score: Mean $(S D)=3.01(0.98)$, Median $=2.97$, Range $=4.42-1.82$.

In general, Spearman correlations between each of the subscales and total scores were all statistically significant. For the 'attitude towards utilization of EBP' $(r=0.48, \mathrm{P}<0.001)$, for the 'skills in EBP $(r=0.83, p<0.001)$ and for the 'use of knowledge source associated with EBPQ' $(\mathrm{r}=0.82, \mathrm{P}<$
$0.001)$. The relationship between attitude and skill( $\mathrm{r}=0.27, \mathrm{P}<$ $0.001)$, attitude and source of knowledge $(\mathrm{r}=0.23, \mathrm{P}<0.001)$ and skill and source of knowledge $(\mathrm{r}=0.46, \mathrm{P}<0.001)$. For more information see table 5 .

Table 5. Correlation between sub scales with total EBPQs.

\begin{tabular}{|c|c|c|c|c|}
\hline Scale and sub scales & attitude & skill in EBP & source of knowledge & Total EBPQ \\
\hline attitude & 1.000 & & & \\
\hline skill in EBP & $.265^{* *}$ & 1.000 & & \\
\hline source of knowledge & $.234^{* *}$ & $.462^{* *}$ & 1.000 & \\
\hline Total EBPQ & $.480^{* *}$ & $.827^{* *}$ & $.819^{* *}$ & 1.000 \\
\hline
\end{tabular}

${ }^{* *}$ Correlation is significant at the 0.01 level (2-tailed)

Note that: All two asterisks $\left(\mathrm{a}^{* *}\right)$ Shows each variables were correlated at $\mathrm{P}<0.001$

Additionally, the reliability of overall questionnaire was evaluated by using Cronbach's alpha coefficient $(\alpha=0.88)$. As can be seen, the value of Cronbach's alpha coefficient is exceeded the accepted criterion of 0.70 for all subscales, in self-reported attitudes, skills and use of knowledge sub scale (see table 6) 
Table 6. A total mean score and Standard Deviation (SD) of EBPQ sub scale with its Cronbach's alpha coefficient.

\begin{tabular}{lll}
\hline Scale or Sub scales & Mean \pm SD & Cronbach's alpha coefficient \\
\hline Self-reported attitude & $3.53 \pm 0.97$ & 0.76 \\
Self-reported skills & $2.82 \pm 1.34$ & 0.86 \\
Source of knowledge & $3.01 \pm 0.98$ & 0.82 \\
Total score & $3.12 \pm 1.05$ & 0.88 \\
\hline
\end{tabular}

\section{Discussion}

To the authors' knowledge, this was the first study conducted in Hunan province south China to examine perceived attitudes towards, skills in and use of knowledge source for implementation of EBP among registered nurses deployed at teaching and referral hospital. With a high level of support from the nurse managers and supervisor in the delivery of the study questionnaires, an exceptionally high response rate $(100 \%)$ was achieved in this study.

Generally, our results suggest that registered nurses who participated in this study held favorable attitudes towards utilization of evidence based practice However, the level of skills in acquiring research evidence and use of evidence to support their decision concerning care of patient were reported as poor.

\subsection{Attitude Towards Utilization of EBP}

In order to assess nurses' perceived attitude towards utilization of EBP, a set of five items were used. While our study findings generally shows that most of the respondents perceived their attitude as favorable. This finding is consistent with study finding conducted in USA, by Williamson, K. M. et al 2015 [3], reported that a registered nurses had positive attitude towards utilization of EBP than their skill. However, even though attitudes towards utilization of EBP were generally favorable in our study participants, misconceptions regarding work load to use up to-date a new evidence appear to persist. Nearly two third (74\%) of participants were agreed with the statement "My workload is too high to keep up-to-date with all new evidences". Similar study has previously been presented in Singapore [15] by using similar instrument. In addition, our study finding is consistent with the finding reported by (Adamu and Naidoo, J. 2015) in which a large percentage $(59.4 \%)$ of the respondents agreed that their workload is too high to keep up to date themselves with all new evidence [40].

In fact, nurse are too busy with high patient load, heavy workloads and insufficient time to use research finding as evidence for their daily care Practice. In addition to this, the hospital organizational cultures are not allow for nurses to search or read research articles during work-hours [45]. Further, in our study, the majority $(61.2 \%)$ of the participants did not preferred to use more traditional methods instead of changing to new approaches. This may imply that nurses need to shift from traditional practices to evidence-driven health care services. This finding is consistent with the study reported from Nigeria in which $(54.1 \%)$ of the participants disagreed that they prefer using more traditional methods instead of changing to new approaches [40]. However, this finding is not coincide with the study finding reported by Farokhzadian, J., et al.,2015 in which the highest number of nurses were preferred to use more traditional methods instead of changing to new approaches [7]

\subsection{Self-Reported Skills in Performing Different EBP Activities}

Although, a majority of nurses agreed with the positive aspects of EBP, most respondents considered themselves to have a poor skills in most areas of evidence-based practice, in particular, reading a research report and having a general notion about its strength and weaknesses $(66.1 \%)$, Evaluating the application of intervention and identify areas of improvement (62.9\%), and Using check list to assess research article $(60.1 \%)$. This result is supported by several study conducted on the assessment of nurses' knowledge, attitude and Practice towards Evidence based practice. As reported, a majority of nurses had low level of skill in performing different EBP activities. For example in study reported (by Heydari A.et al, 2014), the participant's level of Skill in EBP were found to be low [46]. Besides, This result is in line with previous study conducted in Norway by Stokke, K., et al [9] who reported that most nurse had a positive attitude towards evidence-based practice, but the level of self-reported skill in EBP was found to be low extent.

Furthermore, the study from Australia is also in agreement with this finding [47] in which most respondents rated low level of their skill (self- efficacy) in performing deferent EBP activities. Besides, Kang, Y. and I.-S. Yang 2016, in their study explained that the implementation of evidence based practice was only $17.1 \%$. The authors also identified as a problem to be solved: level of searching skills for literature and degree of understanding evidence based practice had statistically significant influences on nurses to implement evidence based practice [45]. This implies that lack of skill or self-confidence is one of the main factor for influencing nurses to use and implement different EBP activity. Hence such problem may affects the quality of health care service in different area of health care institution.

Moreover, the finding of our study indicates that most of participants $(66.1 \%)$ have perceived low level of skill in reading a research report and having a general notion about its strength and weaknesses. This finding is supported by a study conducted on Iranian nurses indicating that a majority of nurses had poor skill in reading a research report and having a general notation about its strength and weakness [7].

Additionally, study conducted in Australia (Fairbrother, G., et al., 2015), stated that the technical skill level including ability to read and understand evidence was found to be low among nurses [48]. This problem or challenges may be due to langauge barrier and lack of ability to understand statistical terms and statistical analysis as well. For instance, (Kang, H., 2015) conducted a research in South Corea [49], and found that majority of participants rated themselves as having a poor skills in undesstanding and using a research reports being written in English language. Besides, having in adequate skill 
in conducting research activity may be one of a barrier to nurses. For example the study conducted by Gerish et al (2011) found that lack of undertaking research is one of the best area in which the particpants identified themselves as novice [50]. Similarly, a recent study conduted by Kang, Y. and I.-S. Yang 2016, found that two-thirds of korean registered nurses did not clearly understand Evidence based practice and most did not regularly read research articles [45]

It is clear that, lack of skill in implementing EBP, and a failure to apply it when making decisions, may lead to the delivery of suboptimal or even ineffective programs, services, and supports, poor patient outcomes, and cost-ineffectiveness. Moreover, Pravikoff et al, reported that registered nurses' had poor skills in performing several EBP: paricularlly, limited computer skills, difficulty in understanding research articles, lack of search skills, and lack of skills to critique or synthesize the literature were cited as a major barriers influencing nurse to use EBP [51]

Further, this study data also suggest that a large proportion of nurses $(77 \%)$ repored their level of skill and confidence as good/very good in Identifying the main clinical issue/ problems. This means nurses had more confident in identdifying and diangosing any clinical issue including the sign and symtom of disease. This is supported by study conducted in Nageria [40], Singapore [15] and Iran [7], the majority of nurses reoprted their higher level of skill in Identifying the main clinical issue/ problems. Moreover, in our study, nurses with higher educational level and longer work experience were reported more confident on their skills in implementing different EBP activities.

\subsection{Utilization of Knowledge Source for Supporting Practice}

The result of our study shows that nurses mainly use experience based knowledge/information resource from human resource (ward colleagues, nursing supervisors and doctors) than evidence gained from printed information source (research journal) and electronic information resource (Medical databases, online tutorials provided by professional associations, medical libraries, foreign hospitals and nursing e- books,). More than half of participants (55.8\%) did not use research journals. These finding is consistent with study conducted in Norway [52], which reported that a large number of nurse used experienced-based knowledge, colleagues and other collaborators for supporting their daily practice than evidence from research journal article.

Moreover, our study finding is similar with the previous study finding reported from Italy [19] which found that Only $41.4 \%$ of nurses often/always read scientific journals or guidelines, $37 \%$ often/always incorporate EBP into practice. Similar study finding from Poland (Jarosława Belowska, et al. 2016) also reported that nearly $42 \%$ of nurses use scientific evidence more often in their everyday nursing practice [20]. Another study conducted in Ethiopia (Hadgu et al. 2015) reported that Only $15.7 \%$ use EBP in their clinical practice always [23].

However, several studies have reported that nurses are expected to establish and make use of the best research evidence to enhance their clinical effectiveness of patient care and improve patients' health outcomes [53]. Nurses must include the latest evidence in their daily decision- making. It is clear that without current best evidence, practice becomes rapidly outdated to the potential detriment of patients. Therefore, nurse must take seriously implementing practices not based on sound evidence [51].

This Study also noted that $(57.1 \%)$ of participants did not use medical databases (e.g. CINAHL). Some of the barriers may be that due to lack of time for literature searching, lack of awareness in using medical databases and lack of skill for conducting online search using different databases. This finding is supported by previous study finding conducted in Finland [54] and Iran [41] in which a large number of nurse lack information retrieval Skills and awareness of searching and using literature as well. However, our result is not coincide with a recent comparative study conducted by (Upton, P., et al., 2015) on nursing professionals working in US and UK [38] in which both nurses working in an academic faculty and clinical faculty frequently use all source of EBP. This may be due to the level of nurse's awareness and available resource in USA, UK and China is not the same. On other hand, the level of utilizing evidence-based practice progress in nursing profession is not well developed in china [24]. Some reasons for the slow growth may be lack of knowledge and skills in EBP, lack of information on EBP, poor access to electronic databases, limited research capacity, and lack of time to do EBP related activities can undermine Chinese nurses. Therefore, the evidence-based practice movement in China still has a long way to go by continuously using scientific evidence in daily care practice.

Further the result of our study indicate that the majority of nurses frequently/always need information from their ward colleagues $(78.7 \%)$, supervisors $(77.6 \%)$ and nursing management staff $(55.7 \%)$. This finding shows that the majority of nurses do not search for scientific research. Rather they seek information from their ward colleague and supervisors who have better knowledge and skill in performing different EBP activities. This finding is supported by a recent study conducted in Sweden by Jansson, I. and A. Forsberg (2016) Nurses and managers had different approaches towards the use of scientific knowledge. Commonly, nurses' use clinical experience and learning from each other and their work as facilitators was structured by managers [55]. This indicates that nurses seek answers to clinical questions on a daily basis. However, the source of knowledge or information they need for evidence is mostly based on sound evidence rather than searching and reading evidence from printed and electronic information source for implementation of evidence based practices.

\section{Limitation of the Study}

Strengths of this study includes the use of a validated and reliable instrument of EBPQ to measure the participant's attitudes, skills and use of knowledge source on 
implementation of EBP. However, some limitations of this study should be considered.

First, the use of a self-reported questionnaire, which is considered as the most convenient data collection method, is difficult to avoid response biases due to misunderstanding of the questionnaire items, or to the participants responding according to what they perceived to be socially desirable.

Second, this study is conducted only in one teaching hospital so the conclusion of this study is not generalizable to other settings. However, the level of education, focus of practice, sampling method, number of participant (when compared with other study area, in other country), the response rate and policy of hospital administration in all Chinese province indicating that our sample was likely to be representative of Chinese nurse.

\section{Conclusion and Recommendation}

\subsection{Recommendation}

Utilization of EBP is the initial step in providing high quality and very effective patient care. On the basis of findings from the current study, support at individual and organizational level are needed to create awareness in continuously utilizing EBP from different source of information rather than only using human information source. This can be created by including a number of registered nurses in participating research mentors and advisors to promote engagement in research among nurses. In addition, provision of funding from both governmental and non-governmental organization is needed to initiate and support new nursing research project for promoting utilization of EBP among Chinese nurses at the work place. Especially more emphasizes should be placed on providing advanced education and training for nurses to improve their skill and confidence in performing different EBP activities.

Another strategy to increase and develop the culture of EBP in Chinese nursing profession is establishing Chinese nursing Journal club. This can be encourage nurses' interest in conducting online search, reading journal article from printed and electronic source to understand all components/elements of EBP. Thus, experiences gained through this strategy can be applied to practice with more confidence. Besides, creating a team for studying scientific finding among nurses may encourage them to share research ideas and engage in group of research projects to improve practice.

However, most nurses working in Chinese hospital have poor access to the library and different source of websites. For example, Google Scholar, YouTube, Instagram and face books are not available in China. This can be undermined nurses to use and read internationally published literature in English language. Thus, nurses in china are facing a number of challenge in understanding research journal published in English language. Therefore more support is needed from Chinese governmental and nursing administrators to provide the resources necessary to encourage utilization of EBP among nurses. On other hand support from educator and supervisor are needed to improve nurses' skill, attitude and utilization of EBP as a basis for quality patient care.

\subsection{Conclusions}

This study clearly shows a meaningful contribution to understand the perceptions of Chinese nurses on utilization and implementation of evidence based practice. Implementation of EBP can only be achieved through integration and utilization of all source of knowledge (printed information source, human information source and electronic information source). This systematic approach that utilizes evidence from different source of information in practice is very essential in providing a dignified, safe and compassionate quality health care service. Because nurses are in the frontline in utilizing, integrating and implementing EBP in any health care organization to enhance quality of healthcare service.

Overall, this study suggest that registered nurses who participated in this study held a favorable attitudes towards utilization of evidence based practice. However, their level of skills in performing different EBP activities and use of knowledge to support decision concerning care of patient were found to be low. However, this conclusion is drawn based on only one healthy organization. Further research is needed to identify the influence of individual and organizational factors on implementation of evidence based nursing practice. Nurse and other healthcare providers must take seriously implementing practices not based on sound evidence. When practices are not based on sound evidence, the physical, emotional and financial cost to patients and their family members can be successfully achieved.

\section{References}

[1] Melnyk et al, The Establishment of Evidence-Based Practice Competencies for Practicing RN and Advanced Practice Nurses in Real-World Clinical Settings: Proficiencies to ImproveHealthcare Quality, Reliability, Patient Outcomes, and Costs. Worldviews on Evidence-Based Nursing,, 2014. 11 (1): p. 5-15.

[2] Odell, E. and K. Barta, Teaching evidence-based practice: the Bachelor of Science in nursing essentials at work at the bedside. J Prof Nurs, 2011.27 (6): p. 370-7.

[3] Williamson, K. M., et al., Utilization of evidence-based practice knowledge, attitude, and skill of clinical nurses in the planning of professional development programming. J Nurses Prof Dev, 2015. 31 (2): p. 73-80.

[4] Robin P. Newhouse, e., Johns Hopkins Nursing Evidence-Based Practice Model and Guidelines. Book Review, 2007: p. 83-85.

[5] Alba DiCenso, et al., Evidence-Based Nursing: A Guide to Clinical Practice. Text book, 2014.

[6] Bostrom, A. M., et al., Factors associated with evidence-based practice among registered nurses in Sweden: a national cross-sectional study. BMC Health Serv Res, 2013. 13: p. 165. 
[7] Farokhzadian, J., et al., Nurse leaders' Attitudes, Self-Efficacy and training Needs for Implementing Evidence-Based Practice: Is It Time for a Change toward Safe Care? Br J Med Med Res, 2015. 7 (8): p. 662-671.

[8] Ubbink DT.; et al., Framework of policy recommendations for implementation of evidence-based practice: a systematic scoping review. B Med.Journal, 2013. 3.

[9] Stokke, K., et al., Evidence based practice beliefs and implementation among nurses: a cross-sectional study. BMC Nursing, 2014. 13 (8).

[10] HoffmannT Bennett S \& Del Mar C, Evidence-Based Practice Across the Health Professions. Text book, 2013: p. http://evolve.elsevier.com/AU/Hoffmann/evidence/.

[11] Raj Subramaniam P. Siraja KC\& Sarita P, Barriers and Facilitators of Utilizing Research Among Nurses in Nepal. J Contin Educ Nurs, 2016. 47 (4): p. 171-9.

[12] Titler, M. G., Translation science and context. Res Theory Nurs Pract, 2010. 24 (1): p. 35-55.

[13] International Council of Nurses, G., Switzerland, Nursing research: a tool for action. Fact sheet, 2009. 2nd edititon p. 1-3.

[14] Saunders, H. and K. Vehviläinen-Julkunen, The state of readiness for evidence-based practice among nurses: An integrative review. International Journal of Nursing Studies, 2016. 56: p. 128-140.

[15] Majid, S., et al., Adopting evidence-based practice in clinical decision making: nurses' perceptions, knowledge, and barriers. J Med Libr Assoc, 2011.99 (3): p. 229-36.

[16] Yoder, L. H., et al., CE: Original Research: Staff nurses' use of research to facilitate evidence-based practice. Am J Nurs, 2014. 114 (9): p. 26-37; test 38; 50.

[17] WHO, Evidence-ledobstetric care Geneva, Switzerland.. 2005: p. 28-30.

[18] Breimaier, H. E., R. J. Halfens, and C. Lohrmann, Nurses' wishes, knowledge, attitudes and perceived barriers on implementing research findings into practice among graduate nurses in Austria. J Clin Nurs, 2011. 20 (11-12): p. 1744-56.

[19] Filippini, A., et al., Evidence-based practice among nurses in Italy. Eval Health Prof, 2011. 34 (3): p. 371-82.

[20] Jarosława Belowska, et al., Analysis of attitudes of nurses towards using scientific literature in everyday clinical practice. Conference Paper, 2016: p. 3596-3603.

[21] McInerney, P. A., Evidence-Based Nursing and Midwifery: The State of the Science in South Africa. 2004: p. 207-208.

[22] Kyalo Mutisya, A., A. KagureKarani, and C. Kigondu, Research Utilization among Nurses at a Teaching Hospital in Kenya. J Caring Sci, 2015.4 (2): p. 95-104.

[23] Hadgu, G., Assessment of Nurses' Perceptions and Barriers on Evidence Based Practice in Tikur Anbessa Specialized Hospital Addis Ababa Ethiopia. American Journal of Nursing Science, 2015. 4 (3): p. 73.

[24] Chen, Y. C., L. C. Tang, and S. S. Chou, [Strategy for promoting evidence-based nursing practice in hospital]. $\mathrm{Hu} \mathrm{Li}$ Za Zhi, 2013. 60 (5): p. 25-30.
[25] Zhou, X., et al., Development of traditional Chinese medicine clinical data warehouse for medical knowledge discovery and decision support. Artif Intell Med, 2010. 48 (2-3): p. 139-52.

[26] Du, Z., et al., Usual source of care and the quality of primary care: a survey of patients in Guangdong province, China. International Journal for Equity in Health, 2015. 14 (1).

[27] Nundy, M., Challenges to Health Service System in China: Institutional and Financial Reforms. 2016: p. 9-23.

[28] Youing Li, X. S., Li Wang,, Evidence-Based Medicine in China International Society for Pharmacoeconomics and Outcomes Research., 2008. 11: p. 156-164.

[29] Barber, S. L., et al., The hospital of the future in China: China's reform of public hospitals and trends from industrialized countries. Health Policy Plan, 2014. 29 (3): p. 367-78.

[30] Jiang, H., et al., Nurses' roles in direct nursing care delivery in China. Applied Nursing Research, 2015. 28 (2): p. 132-136.

[31] Yi-Hao Weng, et al., Increasing utilization of Internet-based resources following efforts to promote evidence-based medicine: a national study in Taiwan. BMC Medical Informatics and Decision Making 2013. 13 (4).

[32] Cheng, L., S. Feng, and Y. Hu, Evidence-based nursing implementation in Mainland China: A scoping review. Nurs Outlook, 2016.

[33] Wang, L. P., et al., Barriers to and facilitators of research utilization: a survey of registered nurses in China. PLoS One, 2013. 8 (11): p. e81908.

[34] Ubbink, D. T., G. H. Guyatt, and H. Vermeulen, Framework of policy recommendations for implementation of evidence-based practice: a systematic scoping review. BMJ Open, 2013. 3(1).

[35] Melnyk, B. M., et al., The State of Evidence-Based Practice in US Nurses. JONA: The Journal of Nursing Administration, 2012. 42 (9): p. 410-417.

[36] Whall, A. L., M. Sinclair, and K. Parahoo, A philosophic analysis of Evidence-Based Nursing: recurrent themes, metanarratives, and exemplar cases. Nurs Outlook, 2006. 54 (1): p. $30-5$

[37] Slattery, S. C. M. J., Evidence-Based Practice in Nursing:A Guide to successful Implementation. 2006.

[38] Upton, P., et al., The evidence-based practice profiles of academic and clinical staff involved in pre-registration nursing students' education: a cross sectional survey of US and UK staff. Nurse Educ Today, 2015. 35 (1): p. 80-5.

[39] Bernadette Mazurek Melnyk, et al., Sustaining Evidence-Based Practice Through Organizational Policies and an Innovative Model. AJN, 2011. 111 (9): p. 59.

[40] Adamu and Naidoo, J., Exploring the Perceptions of Registered nurses towards Evidence- based practice In a selected general Hospital in Nigeria. Africa Journal of Nursing and Midwifery, 2015. 17 (1): p. 33-46.

[41] Farokhzadian, J., R. Khajouei, and L. Ahmadian, Information seeking and retrieval skills of nurses: Nurses readiness for evidence based practice in hospitals of a medical university in Iran. Int J Med Inform, 2015.84 (8): p. 570-7. 
[42] WHO, Process of Translation and Adaptation of Instruments, Geneva.

http://www.who.int/substance abuse/research tools/translatio n/en]Google Scholar) 2010.

[43] Polit D, B. C., Research Manual for Nursing research: generating and assessing evidence for nursing practice 2008. 9 th edition.

[44] Joubert, G. E., R (ed), Epidemiology: a research manual for South Africa. Cape Town. Oxford University Press., 2007. 2nd edition.

[45] Kang, Y. and I.-S. Yang, Evidence-based nursing practice and its correlates among Korean nurses. Applied Nursing Research, 2016. 31: p. 46-51.

[46] Heydari, A., et al., A study of Iranian nurses' and midwives' knowledge, attitudes, and implementation of evidence-based practice: the time for change has arrived. Worldviews Evid Based Nurs, 2014. 11 (5): p. 325-31.

[47] Ziviani, J., et al., Mapping allied health evidence-based practice: providing a basis for organisational realignment. Aust Health Rev, 2015. 39 (3): p. 295-302.

[48] Fairbrother, G., et al., Evidence based clinical nursing practice in a regional Australian healthcare setting: Predictors of skills and behaviours. Collegian, 2015.
[49] Kang, H., Geriatric hospital nurses' perceived barriers to research utilization and empowerment. Asian Nurs Res (Korean Soc Nurs Sci), 2015.9 (1): p. 65-72.

[50] Gerrish, K., et al., Factors influencing the contribution of advanced practice nurses to promoting evidence-based practice among front-line nurses: findings from a cross-sectional survey. J Adv Nurs, 2011.67 (5): p. 1079-90.

[51] Pravikoff, D. S., A. B. Tanner, and S. T. Pierce, Readiness of U.S. nurses for evidence-based practice. Am J Nurs, 2005. 105 (9): p. 40-51; quiz 52.

[52] Dalheim, A., et al., Factors influencing the development of evidence-based practice among nurses: a self-report survey. BMC Health Services Research 2012. 12 (367).

[53] Makic, M. B. F., et al., Putting Evidence Into Nursing Practice: Four Traditional Practices Not Supported by the Evidence. Critical Care Nurse, 2013.33 (2): p. 28-42.

[54] Koivunen, M., M. Välimäki, and H. Hätönen, Nurses' information retrieval skills in psychiatric hospitals - Are the requirements for evidence-based practice fulfilled? Nurse Education in Practice, 2010. 10 (1): p. 27-31.

[55] Jansson, I. and A. Forsberg, How do nurses and ward managers perceive that evidence-based sources are obtained to inform relevant nursing interventions? - an exploratory study. J Clin Nurs, 2016. 25 (5-6): p. 769-76. 